\title{
Holomorphic Mappings from the Ball and Polydisc
}

\author{
H. Alexander
}

Introduction. The holomorphic self-homeomorphisms ("automorphisms") of the open unit ball $B_{n}$ in $\mathbb{C}^{n}$ have long been known [1] - they are given by certain rational functions which are holomorphic on a neighborhood of $\bar{B}_{n}$ and induce a homeomorphism of the boundary, $b B_{n}$, of the ball. Our first result can be viewed as a local characterization of these automorphisms: For $n>1$, a nonconstant holomorphic mapping into $\mathbb{C}^{n}$ which is defined in a neighborhood of a point of $b B_{n}$ and which maps $b B_{n}$ into itself is necessarily an automorphism, or, more precisely, extends to be an automorphism. We apply this to obtain some information on the as yet unsettled question as to whether every proper holomorphic self-mapping of $B_{n}$ is an automorphism. In particular, we recover (Cor. 1.1) a result of Pelles $([3,5])$.

In the second part, we consider holomorphic mappings from polydiscs. According to a classical theorem of Poincaré, there exists no biholomorphism from the polydisc $U^{2}$ in $\mathbb{C}^{2}$ with the ball $\boldsymbol{B}_{2}$. We obtain some integral formulas which yield a quantitative explanation of this phenomenon.

Finally I wish to acknowledge that the above characterization of automorphisms may have been known to the late Professor Löwner, at least for two complex variables. I want to thank Professors $L$. Bers and $C$. Titus for this information on their oral communication with Löwner.

1. The main result of this section is the following characterization of automorphisms of the unit ball.

Proposition 1.1. Let $F=\left(f_{1}, f_{2}, \ldots, f_{n}\right): \Omega \rightarrow \mathbb{C}^{n}$ be holomorphic on a connected neighborhood $\Omega$ of some point of the boundary $b B_{n}$ of the open unit ball $B_{n}$ in $\mathbb{C}^{n}(n>1)$. Suppose that $\sum_{1}^{n}\left|f_{j}(z)\right|^{2} \equiv 1$ for $z \in \Omega \cap b B_{n}$. Then either $F$ is a constant map or $F$ extends to be an automorphism of $B_{n}$.

As a consequence we have that a proper holomorphic self-mapping of the ball which extends to be holomorphic across a single boundary point in $b B_{n}$ is necessarily an automorphism. In particular, since a rational function is regular on an open dense subset of $b B_{n}$ we get the following result of Pelles (published under the former name Eisen-. $\operatorname{man}[3,5])$.

Corollary 1.1. A rational proper self-mapping of $B_{n}(n>1)$ is an automorphism. 
Proof of Proposition 1.1. We consider two cases.

(i) Suppose $\operatorname{det}(J F) \equiv 0$ on $\Omega \cap b B_{n}$, where $J F$ is the Jacobian matrix of $F$. We show that $F$ is a constant map in this case.

As $\operatorname{det}(J F)$ is holomorphic and $b B_{n}$ has real codimension one, $\operatorname{det}(J F) \equiv 0$ on $\Omega$. Let $r$ be the maximum rank of $(J F)(z)$ for $z \in \Omega$. Then $r$ is also the maximum rank of $J F$ on $\Omega \cap b B_{n}$. Without loss of generality, we suppose $e_{n}=(0, \ldots, 0,1) \in b B_{n} \cap \Omega$ and rank $J F\left(e_{n}\right)=r$. By the implicit function theorem, there is a neighborhood of $e_{n}$ on which one of the $f_{j}$ is functionally dependent on the others: Say $f_{n}(z)=g\left(f_{1}(z), \ldots\right.$ $\left.\ldots, f_{n-1}(z)\right)$ for $z \in \bar{\Omega}^{\prime} \subseteq \Omega$ where $\Omega^{\prime}=B_{n} \cap\left\{z: \operatorname{Re} z_{n}>1-\delta\right\}$ for some $\delta>0$ and $g$ is holomorphic in a suitable domain. Fix $z^{0} \in \Omega^{\prime}$ and let $V$ be the irreducible component of $\left\{z \in \Omega^{\prime}: f_{j}(z)=f_{j}\left(z^{0}\right), 1 \leqq j \leqq n-1\right\}$ which contains $z^{0}$. Then $V$ is a subvariety of $\Omega^{\prime}$ of dimension at least one and so $\bar{V}$ meets $b \Omega^{\prime}$. Since the function $z \rightarrow e^{z_{n}}$ does not attain its maximum on $\bar{V}$ at points where $z_{n}=1-\delta$ we see that $\bar{V}$ meets $b B_{n}$ at some point $z^{1}$. Then $f_{j}\left(z^{0}\right)=f_{j}\left(z^{1}\right)$ for $1 \leqq j \leqq n-1$ and therefore

$$
f_{n}\left(z^{0}\right)=g\left(f_{1}\left(z^{0}\right), \ldots, f_{n-1}\left(z^{0}\right)\right)=g\left(f_{1}\left(z^{1}\right), \ldots, f_{n-1}\left(z^{1}\right)\right)=f_{n}\left(z^{1}\right) .
$$

Hence $\sum_{1}^{n}\left|f_{j}\left(z^{0}\right)\right|^{2}=\sum_{1}^{n}\left|f_{j}\left(z^{1}\right)\right|^{2}=1$. Thus $\sum_{1}^{n}\left|f_{j}\right|^{2} \equiv 1$ on $\Omega^{\prime}$. It is an easy exercise (see [2], p. 155) to see from this that the $f_{j}$ are constant functions, as claimed.

Before giving the second case, we shall prove the following result which will be needed for the value $m=0$; the parameter $m$ occurs in the proposition in order to accommodate a proof by induction. A complex line is a complex linear subspace of $\mathbb{C}^{n}$ of dimension one over $\mathbb{C}$.

Proposition 1.2. Let $f_{1}, \ldots, f_{n} ; f_{n+1}, \ldots, f_{n+m}$ be holomorphic functions on $W=\bar{B}_{n} \cap\left\{z:\left|z_{1}\right| \leqq \delta\right\}$ for some $\delta>0$ with $f_{j}(0)=0,1 \leqq j \leqq n+m$, where $n \geqq 1$ and $m \geqq 0$. Suppose

(a) $z \rightarrow\left(f_{1}(z), \ldots, f_{n}(z)\right)$ is $1-1$ on a neighborhood of $W$,

(b) $\sum_{j=1}^{n+m}\left|f_{j}(z)\right|^{2} \equiv 1$ for $z \in b B_{n} \cap W$,

(c) $f_{n+j}$ is a linear combination of $f_{1}, f_{2}, \ldots, f_{n}$ for $1 \leqq j \leqq m$.

Then each $f_{i} / f_{j}$ is constant on complex lines (intersected with $W$ ).

Proof. By induction on $n$. For $n=1$, the conclusion is clear because of (c). Observe that for $n=1$, (b) becomes vacuous if $\delta<1$.

Now say $n>1$ and assume the proposition for $n-1$. Write $f_{n+j}=\sum_{k=1}^{n} C_{j k} f_{k}, 1 \leqq j \leqq m$ and let $C=\left(C_{j k}\right)$, an $m \times n$ matrix. Define the differential operators $T_{j}=\bar{z}_{n} D_{j}-\bar{z}_{j} D_{n}$ for $1 \leqq j \leqq n-1$ where $D_{k}=\frac{\partial}{\partial z_{k}}$. Note that $T_{j}(\bar{f} u)=\bar{f} T_{j}(u)$ if $f$ is a holomorphic function and therefore 
(A) the $T_{j}^{\text {'s commute and }}$

(B) if $T=T_{j_{1}} T_{j_{2}} \ldots T_{j_{s}}$, then $T=Z_{n}^{s} D_{j_{1}} D_{j_{2}} \ldots D_{j_{s}}+$ terms with coefficients vanishing for $z_{1}=\cdots=z_{n-1}=0$ and so $(T u)\left(0, \ldots, 0, z_{n}\right)$ $=\vec{z}_{n}^{(s}\left(D_{j_{1}} \ldots D_{j_{s}} u\right)\left(0, \ldots, 0, z_{n}\right)$.

Now let $T$ be a product of one or more of the $T_{j}$ 's; each $T_{j}$ is a tangential differential operator on $b B_{n}$ (see [4], p. 31) and therefore so is $T$. As $\sum_{i}^{n+m} \bar{f}_{k} f_{k} \equiv 1$ on $b B_{n} \cap W$, we get $0=T\left(\sum_{k=1}^{n+m} \bar{f}_{k} f_{k}\right)=\sum_{k=1}^{n+m} \bar{f}_{k} T f_{k}$ on $b B_{n} \cap W$. Since $T f_{n+j}=\sum_{k=1}^{n} C_{j k} T f_{k}$ for $1 \leqq j \leqq m$ we get

$$
\sum_{k=1}^{n} X_{k} T f_{k} \equiv 0
$$

on $b B_{n} \cap W$ where $X_{k}=\bar{f}_{k}+\sum_{j=1}^{m} C_{j k} \bar{f}_{n+j}=\bar{f}_{k}+\sum_{s=1}^{n} \sum_{j=1}^{m} C_{j k} \bar{C}_{j s} \bar{f}_{s}$. In matrix terms

$$
\left(\begin{array}{c}
X_{1} \\
X_{2} \\
\vdots \\
X_{n}
\end{array}\right)=\left(I+C^{\imath} \bar{C}\right)\left(\begin{array}{c}
\bar{f}_{1} \\
\bar{f}_{2} \\
\vdots \\
\bar{f}_{n}
\end{array}\right) .
$$

Observe that $I+C^{t} \bar{C}$ is nonsingular as $C^{t} \bar{C}$ is Hermitian positive semidefinite. Therefore since $f_{1}, \ldots, f_{n}$ have a common zero only at the origin by (a), it follows that $X_{1}, X_{2}, \ldots, X_{n}$ also have the origin as their only common zero on $W$.

Since $z \rightarrow\left(f_{1}(z), \ldots, f_{n}(z)\right)$ is $1-1$ on a neighborhood of $W$, the Jacobian matrix $J\left(f_{1}, \ldots, f_{n}\right)=\left(D_{i} f_{j}\right)$ has a nonzero determinant. Hence the cofactors of the last row have no common zeros on $W$. Let $a_{1}\left(z_{n}\right), \ldots, a_{n}\left(z_{n}\right)$ be the restrictions of these cofactors to $z_{1}=0, \ldots, z_{n-1}=0$; $a_{j}$ is a holomorphic function for $\left|z_{n}\right| \leqq 1$.

Now apply (1) $n$ times for $T=T_{1}, T_{2}, \ldots, T_{n-1}$ and for an arbitrary $T$. Setting then $z_{1}=z_{2}=\quad=z_{n-1}=0$ and recalling (B) above, we get, after multiplying by $z_{n}$ (or a power of $z_{n}$ in the last case), a homogeneous system of $n$ equations for $X_{1}, \ldots, X_{n}$ :

$$
\begin{aligned}
& \sum_{1}^{n}\left(D_{1} f_{k}\right) X_{k}=0 \\
& \sum_{1}^{n}\left(D_{2} f_{k}\right) X_{k}=0 \\
& \text { for } \quad\left|z_{n}\right|=1 \quad z_{1}=\cdots=z_{n-1}=0 \\
& \sum_{1}^{n}\left(D_{n-1} f_{k}\right) X_{k}=0 \\
& \sum_{1}^{n}\left(D f_{k}\right) X_{k}=0
\end{aligned}
$$


where $D$ is a product of one or more of the $D_{j}$. As the $X_{k}$ 's have no common zeros for $z_{1}, \ldots, z_{n}$ in this range, it follows that the determinant of the system vanishes. Expanding it on the last row and observing that the cofactors of its last row are exactly those of $J\left(f_{1}, \ldots, f_{n}\right)$ we get

$$
\sum_{k=1}^{n} a_{k}\left(z_{n}\right)\left(D f_{k}\right)\left(0, \ldots, 0, z_{n}\right) \equiv 0
$$

for $\left|z_{n}\right|=1$. As these functions are holomorphic we conclude that (2) holds for $\left|z_{n}\right| \leqq 1$.

If $f$ is an analytic function on $W$, we have for $\left(z^{\prime} ; z_{n}\right) \in W$ where $z^{\prime}=\left(z_{1}, \ldots, z_{n-1}\right)$ :

$$
f\left(z^{\prime}, z_{n}\right)=\Sigma \frac{1}{\alpha !}\left(D^{\alpha} f\right)\left(0 ; z_{n}\right) z^{\prime \alpha}
$$

where $\alpha=\left(\alpha_{1}, \ldots, \alpha_{n-1}\right)$ and $D^{\alpha}=\left(D_{1}\right)^{\alpha_{1}} \ldots\left(D_{n-1}\right)^{\alpha_{n-1}}$. By (2) for $\alpha \neq 0$, $\left|z_{n}\right| \leqq 1$,

$$
\sum_{k=1}^{n} a_{k}\left(z_{n}\right)\left(D^{\alpha} f_{k}\right)\left(0, z_{n}\right) \equiv 0
$$

Multiplying by $z^{\prime \alpha}$ and summing over $\alpha$, we get, for $\left(z^{\prime}, z_{n}\right) \in W$ :

$$
\sum_{k=1}^{n} a_{k}\left(z_{n}\right) f_{k}\left(z^{\prime}, z_{n}\right)=b\left(z_{n}\right)
$$

where $b\left(z_{n}\right)=\Sigma a_{k}\left(z_{n}\right) f_{k}\left(0, z_{n}\right)$. As the $f_{j}$ vanish at the origin, $b(0)=0$. Put $z_{n}=0$ and get

$$
\sum_{k=1}^{n} a_{k}(0) f_{k}\left(z^{\prime}, 0\right) \equiv 0
$$

for $z^{\prime} \in W^{\prime}=\left\{\left(z_{1}, \ldots, z_{n-1}\right) \in \mathbb{C}^{n-1}:\left|z_{1}\right| \leqq \delta\right.$ and $\left.\sum_{1}^{n-1}\left|z_{k}\right|^{2} \leqq 1\right\}$.

We have seen that some $a_{j}(0)$ is nonzero; say $a_{n}(0) \neq 0$. Then, by (3), $f_{n}$ is a linear combination of $f_{1}, \ldots, f_{n-1}$ on $W^{\prime}$. Also (a) implies that $z^{\prime} \rightarrow\left(f_{1}\left(z^{\prime}\right), \ldots, f_{n-1}\left(z^{\prime}\right)\right)$ is $1-1$ on $W^{\prime}$. We can now apply the induction hypothesis to $f_{1}, \ldots, f_{n-1} ; f_{n}, \ldots, f_{n+m}$ on $W^{\prime} \leqq \mathbb{C}^{n-1}$ to conclude that each $f_{i} / f_{j}$ is constant on complex lines in the hyperplane $z_{n}=0$.

By making a small rotation of coordinates and applying the induction hypothesis with a slightly smaller $\delta$, we obtain by the previous argument that each $f_{i} / f_{j}$ is constant on complex lines near the hyperplane $z_{n}=0$. By analytic continuation, each $f_{i} / f_{j}$ is constant on all complex lines.

We can now complete the proof of Proposition 1.1.

(ii) Suppose $J F$ is nonsingular at some point of $\Omega \cap b B_{n}$, say at $e_{1}=(1,0, \ldots, 0)$, with no loss of generality. Then $F$ is $1-1$ on some 
neighborhood of $e_{1}$. Choose $0<r<1$ so that $F$ is $1-1$ on a neighborhood of $\bar{B}_{n} \cap\left\{z \in \mathbb{C}^{n}: \operatorname{Re} z_{1} \geqq r\right\}$. Fix $r<a<1$ and define an automorphism $\varphi$ of $B_{n}$ by $\varphi\left(z_{1}, \ldots, z_{n}\right)=\left(\left(z_{1}+a\right) /\left(1+a z_{1}\right), \quad z_{2} \sqrt{1-a^{2}} /\left(1+a z_{1}\right), \ldots\right.$ $\left.\ldots, z_{n} \sqrt{1-a^{2}} /\left(1+a z_{1}\right)\right)$. As $F$ is defined and $1-1$ on a neighborhood of $\left\{z \in \mathbb{C}^{n}:\|z\| \leqq 1\right.$ and $\left.z_{1}=a\right\}, F \circ \varphi$ is defined and $1-1$ on a neighborhood of $\left\{z \in \mathbb{C}^{n}:\|z\| \leqq 1,\left|z_{1}\right| \leqq \delta\right\}$ for some $\delta>0$. Let $\tau$ be an automorphism of $B_{n}$ for which $\tau\left(F\left(a e_{1}\right)\right)=0\left(F\left(a e_{1}\right) \in B_{n}\right.$ by the maximum principle applied to the subharmonic function $\Sigma\left|f_{j}\right|^{2}$ ). The holomorphic mapping $\tilde{F}=\tau \circ F \circ \varphi$ is such that (1) $\tilde{F}$ is defined and $1-1$ on a neighborhood of $W=\left\{z \circ \mathbb{C}^{n}:\|z\| \leqq 1,\left|z_{1}\right| \leqq \delta\right\},(2) \tilde{F}(0)=0$, and (3) $\|\tilde{F}(z)\|=1$ if $z \in W \cap b B_{n}$. If we can prove that $\tilde{F}$ extends to be an automorphism of $B_{n}$, it is clear that the same will be true for $F$. Thus, without loss of generality, we may assume that (1), (2), and (3) hold for $F$ in place of $\tilde{F}$.

We apply Proposition 1.2 to $f_{1}, \ldots, f_{n}$ and conclude that each $f_{i} / f_{j}$ is constant on complex lines. The zero set of $f_{j}$ is a set of complex lines. For if $p(\neq 0) \in W$ and $f_{j}(p)=0$, then, as $z \rightarrow\left(f_{1}(z), \ldots, f_{n}(z)\right)$ is $1-1$ on $W$, there is $k$ such that $f_{k}(p) \neq 0$; as $f_{j} / f_{k}$ is constant on the complex line through $p$ and equal to zero at $p$ itself, $f_{j} \equiv 0$ near $p$ on this complex line and so $f_{j} \equiv 0$ on the entire complex line (intersected with $W$ ). Since $J F(0)$ is nonsingular, $\left(d f_{j}\right)(0) \neq 0$. It follows that each $f_{j}$ is of the form $f_{j}=k_{j} \psi_{j}$ on $W$, where $k_{j}$ is a linear homogeneous polynomial and $\psi_{j}$ is a nowhere vanishing analytic function on $W$. Next we observe that the functions $\psi_{i} / \psi_{j}$ are constant on complex lines and so are all constant multiples of a single function. Thus $f_{j}=h_{j} \psi$ on $W$, where $h_{j}$ is a linear homogeneous polynomial and $\psi$ is non-vanishing on $W$. Let $L$ be a fixed complex line close enough to the hyperplane $z_{1}=0$ so that $L \cap W$ is a $\operatorname{disc} \Delta$ of radius one in $L$; i.e., $b \Delta \leqq b B_{n}$. Then $\Sigma\left|f_{j}(z)\right|^{2} \equiv 1$ on $b \Delta$. As the $\left|h_{j}\right|$ are constant on $b \Delta$, we conclude that $|\psi|$ is constant on $b \Delta$. Being nonvanishing on $\Delta, \psi$ is constant on $\Delta$. Hence $\psi$ is constant on $W$. Therefore $F$ is the restriction to $W$ of a (complex) linear transformation of $\mathbb{C}^{n}$. Since $\|F(z)\|=1$ for $z$ on an open subset of $b B_{n}$ it follows that $F$ is (a restriction of) a unitary linear transformation.

Q.E.D.

Remark. We have assumed that $F$ is holomorphic across a point of the boundary but it is clear from the proof that we need only have assumed that $F$ be $C^{\infty}$ up to the boundary and holomorphic in the interior.

2. We shall now consider bounded holomorphic functions on the polydisc $U^{n}=\left\{z \in \mathbb{C}^{n}:\left|z_{1}\right|<1, \ldots,\left|z_{n}\right|<1\right\}$. The topological boundary of $U^{n}$ is made up of $n$ pieces each of which is a product of a unit circle and $n-1$ unit discs and thus carries a natural induced surface measure of total mass $2 \pi(\pi)^{n-1}$. We let $\sigma$ be the normalized surface measure on $b U^{n}$; i.e., $\sigma=\left(2 \pi^{n} n\right)^{-1} \mu$ where $\mu$ is surface (i.e., Hausdorff $2 n-1$ ) measure on $b U^{n}$, so that $\sigma\left(b U^{n}\right)=1$. Haar measure on the torus $T^{n}$ will be denoted 
by $m$. We recall that a holomorphic function $f=\Sigma a_{\alpha} z^{\alpha}$ on $U^{n}$ is in the Hardy space $H^{2}\left(U^{n}\right)$ ([8]) if $\|f\|_{2}=\left(\Sigma\left|a_{\alpha}\right|^{2}\right)^{1 / 2}<\infty$.

Proposition 2.1. Let $f \in H^{2}\left(U^{n}\right)$ with $f(0)=0$. Then

$$
\int_{b U^{n}}|f|^{2} d \sigma \leqq \frac{n+1}{2 n} \int_{T^{n}}|f|^{2} d m \text {. }
$$

Proof. Implicit in the assertion is the fact that the boundary value function of $f$ on $b U^{n}$ exists in the $L^{2}$ sense for the measure $\sigma$. Since the functions which are holomorphic on $\overline{U^{n}}$ are dense in $H^{2}\left(U^{n}\right)$, it will suffice to verify (1) for such functions. Let $S_{n}$ be the part of $b U^{n}$ given by $\left|z_{1}\right| \leqq 1, \ldots,\left|z_{n-1}\right| \leqq 1,\left|z_{n}\right|=1$. Let $\lambda$ be planar Lebesgue measure. Then

$$
\begin{aligned}
\int_{S_{n}}\left|f\left(z_{1}, \ldots, z_{n-1}, e^{i \theta}\right)\right|^{2} d \lambda\left(z_{1}\right) \ldots d \lambda\left(z_{n-1}\right) d \theta \\
=\Sigma\left(\int\left|z^{\alpha}\right|^{2} d \lambda\left(z_{1}\right) \ldots d \lambda\left(z_{n-1}\right) d \theta\right)\left|a_{\alpha}\right|^{2} \\
=\Sigma \frac{2 \pi^{n}\left|a_{\alpha}\right|^{2}}{\left(\alpha_{1}+1\right) \ldots\left(\alpha_{n-1}+1\right)}=\Sigma \frac{2 \pi^{n}\left(\alpha_{n}+1\right)\left|a_{\alpha}\right|^{2}}{\left(\alpha_{1}+1\right) \ldots\left(\alpha_{n}+1\right)} .
\end{aligned}
$$

A similar formula holds for the $n-1$ other faces of $b U^{n}$, adding these and normalizing, we get

$$
\int|f|^{2} d \sigma=\frac{1}{n} \Sigma \frac{\left(\alpha_{1}+\alpha_{2}+\cdots+\alpha_{n}+n\right)}{\left(\alpha_{1}+1\right) \ldots\left(\alpha_{n}+1\right)}\left|a_{\alpha}\right|^{2} .
$$

As $f(0)=0,|\alpha|=\alpha_{1}+\cdots+\alpha_{n}>0$ in this sum and so

$$
\left(\alpha_{1}+\cdots+\alpha_{n}+n\right) /\left(\alpha_{1}+1\right) \ldots\left(\alpha_{n}+1\right) \leqq(|\alpha|+n) /(|\alpha|+1) \leqq(1+n) / 2 .
$$

Therefore we get $\int|f|^{2} \mathrm{~d} \sigma \leqq(n+1) / 2 n \Sigma\left|a_{\alpha}\right|^{2}=(n+1) / 2 n \int|f|^{2} d m$.

Remark. If we do not assume that $f(0)=0$, we can apply (1) to $f-f(0)$ and obtain

$$
\int|f|^{2} d \sigma \leqq \frac{n-1}{2 n}|f(0)|^{2}+\frac{n+1}{2 n} \int|f|^{2} d m .
$$

Here we have used the fact that $\int f d m=f(0)=\int f d \sigma$.

Put $\|f\|_{\infty}=\sup \left\{|f(z)|: z \in U^{n}\right\}$ for bounded $f$ on $U^{n}$. Since $\|f\|_{2} \leqq\|f\|_{\infty}$ we have

Corollary 2.1. If $f \in H^{\infty}\left(U^{n}\right)$ and $f(0)=0$, then

$$
\int|f|^{2} d \sigma \leqq \frac{n+1}{2 n}\|f\|_{\infty}^{2} .
$$

This corollary implies, for $n>1$, that there is no non-constant bounded holomorphic function $f$ on $U^{n}$ with $\|f\|_{\infty}=1$ and $|f|=1, \sigma$ a.e. on $b U^{n}$. (If such a function $f$ existed, we could obtain one vanishing 
at the origin: $(f-f(0)) /(1-\overline{f(0)} f))$. Whether such a function ("an inner function") exists on the unit ball $(n>1)$ is an interesting open question (cf. [7]). This question could be answered negatively if an analogue to Corollary 2.1 - with $(n+1) / 2 n$ replaced by any constant less than one could be proved for the ball ( $d \sigma$ would be normalized surface measure on $b B_{n}$ ).

For $F: \Omega \rightarrow \mathbb{C}^{s}$ with $F=\left(f_{1}, \ldots, f_{s}\right)$, we let $\|F\|(z)=\left(\sum_{1}^{s}\left|f_{j}(z)\right|^{2}\right)^{1 / 2}$.

Corollary 2.2. Let $F: U^{n} \rightarrow B_{k}$ be a holomorphic map. Then

$$
\int\|F\|^{2} d \sigma \leqq \frac{n+1}{2 n}+\frac{n-1}{2 n}\|F(0)\|^{2} .
$$

Proof. Apply (1)' to each $f_{j}, 1 \leqq j \leqq k$, and add to get

$$
\int\|F\|^{2} d \sigma \leqq(n+1) / 2 n \int\|F\|^{2} d m+(n-1) / 2 n\|F(0)\|^{2} .
$$

Since $\|F\| \leqq 1$, the assertion follows.

Remark. From (2) we conclude that there is no proper holomorphic mapping from $U^{n}(n>1)$ to $B_{k}$; for if $F$ were proper, then $\|F\| \equiv 1$ on $b U^{n}$ would imply that the left side of (2) were one, while the right side is clearly less than one. This gives a quantitative explanation for the nonexistence theorem of Poincaré. An alternate proof of the non-existence of proper maps is given by the methods of $[8]$ (\$ 7.3). More general results on the non-existence of holomorphic covering correspondences have been obtained by Stein and Rischel [9].

Still another way of viewing the Poincare theorem comes from the following $L^{2}$ formula. For a function $f$ defined on $U^{n}$ write $f\left(z_{1} ; 0\right)$ for $f\left(z_{1}, 0, \ldots, 0\right)$ for $\left|z_{1}\right|<1$. Then

Proposition 2.2. Let $F=\left(f_{1}, \ldots, f_{k}\right): U^{n} \rightarrow B_{k}$ be a holomorphic map.

$$
\int_{T^{n}}\left\|F(z)-F\left(z_{1} ; 0\right)\right\|^{2} d m(z) \leqq \frac{1}{2 \pi} \int_{0}^{2 \pi}\left(1-\left\|F\left(e^{i \theta} ; 0\right)\right\|^{2}\right) d \theta .
$$

where the integrands are the a.e. defined boundary functions.

Proof. Since $\int f(z) \overline{f\left(z_{1} ; 0\right)} d m=\int\left|f\left(z_{1} ; 0\right)\right|^{2} d m$ for $f \in H^{2}\left(U^{n}\right)$, we get $\int\left|f_{j}(z)-f_{j}\left(z_{1} ; 0\right)\right|^{2} d m=\int\left|f_{j}\right|^{2} d m-\int\left|f_{j}\left(z_{1} ; 0\right)\right|^{2} d m$. Adding for $1 \leqq j \leqq k$ we obtain $\int\left\|F-F\left(z_{1} ; 0\right)\right\|^{2} d m=\int\|F\|^{2} d m-\int\left\|F\left(z_{1}, 0\right)\right\|^{2} d m$ $\leqq 1-\frac{1}{2 \pi} \int_{0}^{2 \pi}\left\|F\left(e^{i \theta} ; 0\right)\right\|^{2} d \theta=\frac{1}{2 \pi} \int_{0}^{2 \pi}\left(1-\left\|F\left(e^{i \theta} ; 0\right)\right\|^{2}\right) d \theta$.

Remark. From (3) we see that a measure of the independence of $F$ from the variables $z_{2}, \ldots, z_{n}$ (in the $L^{2}$ sense) is given by the closeness of 
$\|F\|$ to one on the boundary of the disc $\left|z_{1}\right|<1, z_{2}=\cdots=z_{n}=0$. In particular, if the restriction of $F$ to this disc is a proper mapping into $B_{k}$, then $F$ is independent of $z_{2}, \ldots, z_{n}$. This can be paraphrased as follows: If $F_{0}: U \rightarrow B_{k}$ is proper and holomorphic, then, viewing $U$ as $U \times\{0\}$ $\subseteq U \times U^{n-1} \subseteq U^{n}$, i.e., as a subvariety of $U^{n}(n>1)$, the only extension of $F_{0}$ to a holomorphic map $U^{n} \rightarrow B_{k}$ is the obvious extension which is independent of $z_{2}, \ldots, z_{n}$. This fact, of course, contains the Poincaré theorem. It is also of interest to compare it to a recent result of Royden [6] who proves that if $F_{0}: U\left(=U \times\{0\} \subseteq U^{n}\right) \rightarrow \Omega$ is a holomorphic embedding, where $\Omega$ is a complex $n$-manifold, then for any $0<r<1$, there exists an extension of $F_{0}$ to a holomorphic embedding $F:(r U) \times U^{n-1} \rightarrow \Omega$. We see that $r<1$ is needed in Royden's theorem.

\title{
References
}

1. Behnke,H., Thullen, P.: Theorie der Funktionen mehrerer komplexer Veränderlichen, Erg. d. Math., III, 3, reprinted by New York: Chelsea Pub. Co.

2. Bochner, S., Martin, T.: Several complex variables, Princeton University Press, 1948

3. Eisenman, D.: Proper holomorphic self-maps of the unit ball. Math. Ann. 190, 298-305 (1971)

4. Hörmander, L.: An introduction to complex analysis in several variables. Princeton New Jersey: Van Nostrand, 1966

5. Pelles, D.: Correction to [3]. Math. Ann. 202, 135-136 (1973)

6. Royden, H.: The extension of regular holomorphic maps. Proc. Amer. Math. Soc., to appear

7. Rubel,L., Shields,A.: The failure of interior-exterior factorization in the polydisc and ball. Tôhoku Math. J. 24, $409-413$ (1972)

8. Rudin, W.: Function theory in polydiscs. New York: Benjamin, 1969

9. Rischel, H.: Holomorphe Utberlagerungskorrespondenzen. Math. Scand. 15, 49-63 (1964)

\author{
H. Alexander \\ Department of Mathematics \\ University of California at San Diego \\ La Jolla, Cal, 92037, USA \\ and \\ University of Michigan \\ Ann Arbor, Mich. 48104, USA
}

